\title{
The Language of Synchronous and Asynchronous Interactions of EMC
}

\author{
Narine Madoyan \\ Yerevan State University
}

Tudying the global village means taking a considerable account of the Netspeak.

The main linguistic peculiarities of Netspeak can be seen in Computer Mediated Communication (CMC) basically in its various types. Herring (2007:1) defines CMC as "predominantly text-based human-human interaction mediated by network computers or mobile telephony". The term CMC does not seem quite appropriate as apart from a computer the interaction can take place through any electronic device like small gadgets, cell phones or palmtops, and due to this many scientists redefined the linguistic term CMC introducing Electronically Mediated Communication (EMC) instead (Crystal 2012).

The language of EMC may differ according to its types. It is common knowledge that EMC can take place in synchronous and asynchronous time. Due to this the communication on the Internet is generally subdivided into two major forms: synchronous and asynchronous communication. In asynchronous communication the conversation does not take place in real time. One of the types of asynchronous communication is the email which is the use of computer systems to transfer messages between users - now chiefly used to refer to messages sent between private Mailboxes. There is a time lag between the sender of the message and the receiver. Analyzing characteristic features of email language many scientists see its similarity with the language of a formal letter (Crystal 2005). E.g.:

\section{Recommendation letter}

Narine, Thank you for your support. I attach the form already filled in. The name, title, position and phone number of the reference I need to fill in myself, please write that info in the email, or I will ask you to do it via phone. So basically I need a signature on the second page of this document and the date. I will take care of the seal myself.

The above email is written in formal style which resembles the language of an ordinary letter as it contains an addressee, a title, a main body and conclusion. In asynchronous communication the sender has time to edit the letter to check the spelling, and in the above-mentioned case it lacks emotional colouring. In her research Baron regarded email as formal written English but did not adhere to the same view as in her book published two years later where she stated that the language of the email does not closely resemble formal written English (Baron 2000:250-259). In this connection it should be mentioned that there are emails which clearly breach all the rules common to the style of letter writing. The title of the following example starts with a special question although the sender of the message and the recipient do not know each other. E.g.: 
Narine, Why didn't you tell me? You never told me you were having trouble making money online.... This is what you've been looking for: It's brand new, it works, and it's free. Enjoy.

(newsletter@realtranslatorjobs.com via xa.icpbounce.com)

The email has a title which is common to a letter, although it doesn't contain the formal style of language.The aforementioned mail can be considered as junk mail since it contains promotional materials. David Crystal states that junk mail doesn't contain any name and senders simply try to persuade you to become a millionaire in a day (Crystal 2005) although in the above example written in active voice the sender uses direct address so that the recipient may think that this is a real opportunity to get money.

The sender of the massage never wants to show that the sender and the recipient do not know each other which gives her the opportunity to give a piece of advice in making money. If you are a newbie (newcomer, especially in computing or on the Internet) in the global village you will probably answer the email.

Thus, analyzing the given example, we can say, although not quite definitely, that the language of the email is mainly formal. We can state that, opposite to the style of email language, the chat has all the characteristic features typical of the oral speech. The communicators interact in real time so the participants are breaking the linguistic conventional norms. As the communication takes place in real time,the communicators break the accepted linguistic norms first of all to save the keystroke. The analysis of the language used in chat rooms shows that there are many orthographic, semiotic novelties, which are used to express the speaker's attitude and emotions towards the utterance. In the following example the orthographic novelties are expressed through letter homophones $r$ for the auxiliary "to be" in present indefinite and $u$ for the personal pronoun "you". The speaker starts the conversation with a rhyme using western emoticons to show his interest in chat rooms.

$<$ Cheeky_Chico $>$ Whether you're skinny, slim or fat .. have a husband, boyfriend or just a cat .. miss universe or ugly like a rat .. Whomever you are, just let's have a chat ${ }^{\wedge}$

$<$ lili_998> nice

$<$ Cheeky_Chico $>$ thanks :)

$<$ Cheeky_Chico $>$ how $r$ ?

$<$ lili_998> fine where ru from

$<$ Cheeky_Chico $>$ im $27 \mathrm{~m}$ spain

$<$ Cheeky_Chico $>u$ ?

If, in online communication, the whole sentence is written in capital letters it means that the speaker is highly excited. Otherwise stated, the high pitch of the voice is expressed by means of capitalization. E.g.:

$<\mathrm{d}$-ten $>H I L U L U$

$<$ Twillight46_>hb 
The acronym $h b$ in the above-mentioned example indicates to something "highly beautiful". The frequent use of abbreviations and acronyms in chat rooms aims at saving the keystrokes, but it is also used as a code language for certain social groups. According to Bodomo acronyms are divided into

- Sentence acronyms (GTG (got to go), BRB (be right back), LOL (laughing out loud)

- Acronyms of letter homophones (U (you), $R$ (are)

- Combination of letter and number homophones (B4 (before)

- Reduction of individual words (tml (tomorrow), coz (because), gdnite (good night)

The analysis of chat language testifies to the frequent usage of acronyms by representatives of numerous and different chat rooms. E.g.:

$<$ Pecheur $>$ hey there lulu

$<$ BlackForestMary $>b r b$ (sentence acronym)

lulu>where $\boldsymbol{r} \boldsymbol{u}$ from (acronyms of letter homophones)

$<$ crystal69>lolb4katrina (combination of letter and number homophones)

$<$ redmanredtemper $>u$ got me

$<$ redmanredtemper $>$ cos people think raey is a girly girl (reduction of individual words)

In the illustrated examples we can see that the participants of the conversation are using various types of acronyms. The analysis has come to prove that the language of a chat is unique and widely applies grammatical, semantic, semiotic, orthographic novelties which tend to imitate the spoken English language.

\section{References:}

1. Baron, N. (2003) Language of the Internet. // The Standford Handbook for Language Engineers. / Ed. by A. Farghali. Standford: CSLI Publication.

2. Bodomo, A.B. (2010) Computer-Mediated Communication for Linguistics and Literacy. USA Information Science Reference (an imprint of IGI Global).

3. Crystal, D. (2001) Language and the Internet. Cambridge: CUP.

4. Crystal, D. (2012) Internet Linguistics. / Lectures at British Council: Yerevan, Armenia.

5. Thurlow, C.; Lengel, L. \& Tomic, A. (2004) Computer Mediated Communication: Social Interaction and the Internet. London: SAGE.

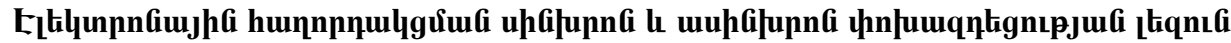

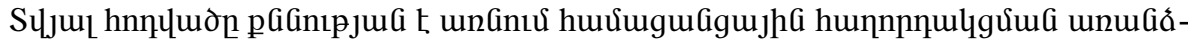

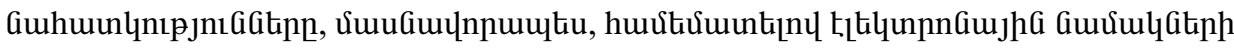




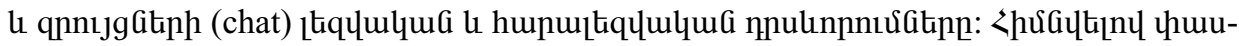

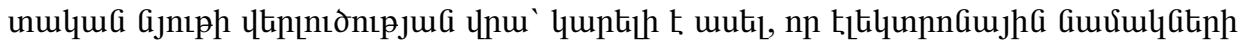

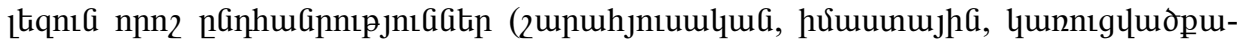

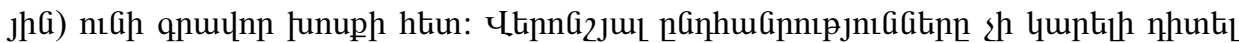

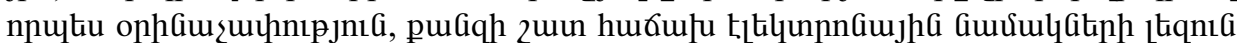

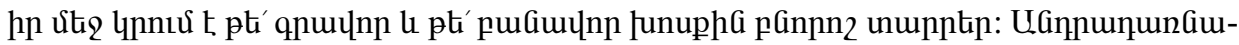

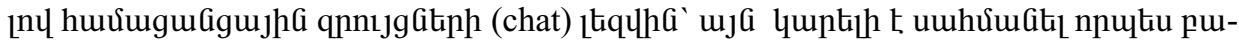

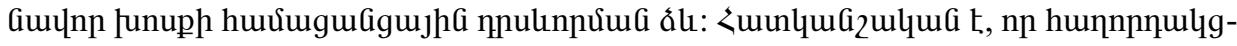

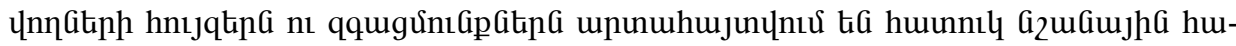
ưulumph ưhongny: 